\title{
Sand Dune Hurdles
}

\section{A simple experimental setup allows researchers to pinpoint the conditions under which a migrating sand dune crosses over an obstacle or gets trapped against it.}

By Matteo Rini

S and dunes form when loose grains of sand are moved by wind or water. As they migrate, these dunes can bury obstacles along their path, endangering infrastructures such as roads and underwater pipelines. Despite the great potential hazards, there aren't established models to predict what happens when sand dunes encounter obstacles. Now, Karol Bacik of the University of Cambridge, UK, and co-workers have studied, in a simplified two-dimensional setup, the dynamics of subaqueous dunes [1]. They show that the outcome of the dune-obstacle interaction-whether the dune crosses over the obstacle and continues on its way or gets trapped against it-can be predicted by analyzing how the fluid flows around the obstacle before the sand starts piling up. The results could help in developing fast, data-driven diagnostic tools for forecasting the impact of dunes on human infrastructures.

The researchers' setup consists of an annular channel where water is driven by submerged paddles. The sand is mimicked by glass beads on the channel floor, while the obstacles are
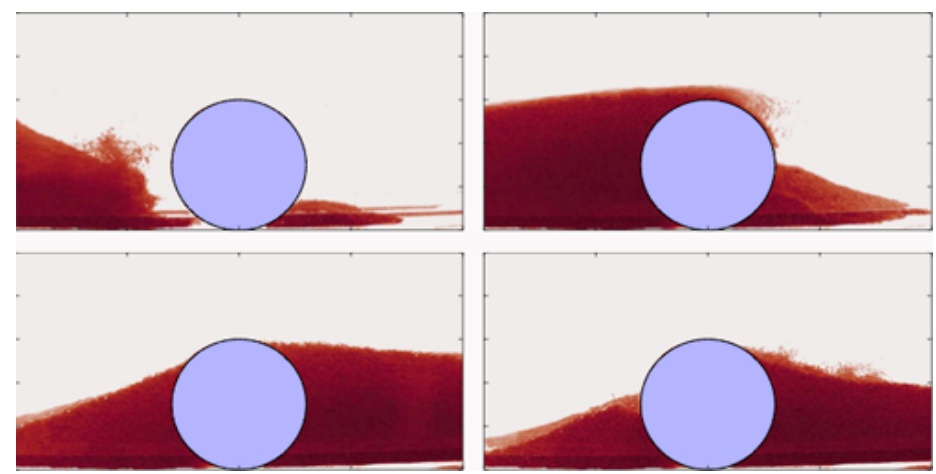

Credit: K. Bacik et al. [1] polystyrene cylinders and prisms. The researchers observed that dune trapping and crossing were determined by the flow velocity and the size and shape of the obstacles. The main result, they say, is that the dune dynamics can be related to the flow patterns in the proximity of the obstacle.

The researchers acknowledge that the results aren't directly applicable to real dunes in three dimensions-in the setup, the dunes that form in the narrow ring are two dimensional-nor to wind-blown dunes like those found in deserts. However, they say that those more complex situations may be tackled by numerical simulations, for which their experiments could serve as a validation benchmark.

Matteo Rini is the Editor of Physics.

\section{REFERENCES}

1. K. A. Bacik et al., "Dynamics of migrating sand dunes interacting with obstacles," Phys. Rev. Fluids 6, 104308 (2021). 